\title{
Ontología: ser, lenguaje e historia
}

\author{
Ricardo Guerra Tejada
}

$\mathrm{E}$

n Heidegger, la existencia humana en tanto que "ser-ahí" (Dasein) como ser en el mundo, ser con otros, en su relación originaria con los entes y con el ser, implica la libertad y la finitud como base y camino de la metafísica a la ontología, al ser que se manifiesta (acontece) en el lenguaje y en el tiempo. Tránsito de la ontología fundamental de Sein und Zeit, "Ser y tiempo" (ser de los entes, ontoteología), a la ontología, es decir, al ser y su "acontecer" en el Dasein.

No se trata de un resultado o de un sistema, sino del camino posible en nuestro tiempo, del fin de la metafísica y del nihilismo, de la técnica, la poesía y el pensar.

Heidegger desarrolla en Sein und Zeit su idea de la hermenéutica y la fenomenología como ontología fundamental, es decir, como punto de partida y base para el planteamiento del problema del ser.

El ser del Dasein es base o preparación para la ontología, es decir, el problema del ser. A todo lo largo de Sein und Zeit se insiste constantemente en esto.

Para Heidegger, la ontología fundamental, la analítica existenciaria, la hermenéutica, la fenomenología del Dasein como "ser en el mundo" es tránsito a la ontología; a la cuestión del ser.

Independientemente del desarrollo de las investigaciones, cursos, publicaciones, la cuestión es la del ser. Sein und Zeit es camino para la ontología.

Si tomamos lo anterior rigurosamente, podemos comprender el rechazo radical de Heidegger a toda interpretación de su obra como filosofía de la vida, antropología o existencialismo.

En Sein und Zeit se logra la descripción fenomenológica más rigurosa del Dasein, del ser en el mundo, de la existencia humana, pero esto sólo es válido como fundamento o camino de la ontología a la cuestión del ser. En cada capítulo, en cada párrafo, se insiste sin la menor duda en esto. Lo que importa 
para Heidegger es el problema del ser y el del Dasein necesariamente unidos. La cuestión del ser, de la ontología, es lo decisivo y esencial.

Se habla de cambios, de etapas distintas en la obra de Heidegger, pero no sólo las conferencias y los ensayos sino especialmente los cursos (que ahora son accesibles en las Obras completas) confirman la evolución y coherencia de sus ideas. No pensamos que haya "saltos", se trata del desarrollo a lo largo de su vida de su concepción o idea del pensar filosófico. El camino o la primera parte prepara la continuación. En Sein und Zeit se logra establecer el primer punto, el camino de la fenomenología como ontología fundamental, obra de gran valor y riqueza filosófica esencial en el siglo Xx, y ésta es la base para el camino del pensar del ser de la ontología.

En los "cursos" (recién publicados) posteriores a Sein und Zeit se plantea con claridad y rigor lo mismo, la necesidad del tránsito a la ontología, pero sobre la base y a partir de la ontología fundamental.

Quedarse en la etapa preparatoria -que es necesaria-sería negar su sentido último, la cuestión del ser, y aceptar, quizás, coincidencias con el existencialismo, la antropología o las ciencias sociales.

En su obra es imposible encontrar cuestiones distintas a la ontología, al pensar, a la poesía y al arte; sabemos que en lo práctico y político no predominó ni la racionalidad, ni la ética. En ese sentido, Heidegger en una tradición de grandes pensadores que desde Platón se equivocaron en la moral y en la política.

En el curso del verano de 1927 publicado en el tomo 24 de la Gesamtausgabe (1975): "Die Grundprobleme der Phänomenologie" (Los problemas fundamentales de la fenomenología), en la primera página, parágrafo primero "Exposition und allgemeine Gliederung des Themas" (Exposición y desarrollo general del tema), en una nota indica que es una nueva elaboración de la tercera sección de la primera parte de Sein und Zeit (Ser y tiempo).

En el tercer parágrafo de la Introducción, "Philosophie als Wissenschaft vom Sein" (Filosofía como ciencia del ser), sostiene con claridad y precisión: Das Sein ist das echte und einzige Thema der Philosophie.

El ser es el auténtico y único tema de la filosofía y no es invento nuestro, dice Heidegger; este planteamiento surge en el comienzo de la filosofía, en la Antigüedad y culmina en forma grandiosa en la Lógica de Hegel. Afirmamos ahora que el ser es "el auténtico y único tema de la filosofía".

La filosofía no es ciencia de los entes, sino del ser o con la expresión griega: ontología. La filosofía es ciencia del ser. Las ciencias que tratan del ente son ciencias positivas: la matemática y las ciencias naturales, la historia del arte, de la política y de las religiones. Las ciencias son del dominio o campo de los entes. "Los entes son algo (Seindes -das ist etwas), pero ¿el ser es como la nada? Hegel dijo: Sein und Nichts sind dass selbe. Philosophie als 
Wissenschaft vom Sein die Wissenschaft von Nichts (ser y nada son lo mismo. Filosofía como ciencia del ser la ciencia de la nada.)

¿Qué significa ser? ¿Cómo es posible su comprensión?

Aristóteles escribió en la Metafísica: "Lo que desde antiguo y ahora y en el futuro permanentemente es buscado y donde la pregunta falla siempre de nuevo es en el problema ¿qué es el ser?"1

Cuestión primera, última y fundamental de la filosofía es: ¿Qué significa ser? ¿Cómo comprender algo como el ser? ¿Cómo es posible la comprensión del ser?

Hay algunas tesis esenciales que se discuten en Los problemas fundamentales de la fenomenología (1927):

1. La tesis de Kant: Sein ist kein reales Prädikat (El ser no es predicado real).

2. La tesis de Aristóteles y la escolástica (ontología medieval): Zur Seins verfassung eines Seinden gehören das Was-Sein (essentia) und das Vorhandensein (existentia) (A la estructura del ser de un ente pertenecen: el que es (esencia) y el existir (existencia).

3. La ontología moderna: Die Grundweisen des Seins sind das Sein der Natur (res extensa) und das Sein des Geistes (res cogitans) (Los modos fundamentales del ser son: el ser de la naturaleza (res extensa) y el ser del espíritu (res cogitans)).

4. Las tesis de la lógica, en sentido amplio: Alles Seinde last sich unbeschadet seiner jeweiligen seinsweise ausprechen durch das "ist"; das Sein der Kopula (Todo ente, sin perjuicio de su particular modo de ser, se deja expresar por el "es"; el ser de la cópula).

Se desarrollan estas tesis en la primera parte del curso. Son insuficientes mientras no se responda la pregunta fundamental de toda ciencia del ser: la pregunta por el sentido del ser en general (die Frage nach dem Sinn von Sein überhaupt) y esta cuestión se trata en la segunda parte del curso.

Antes de considerar los puntos fundamentales para comprender esta "elaboración" de lo que faltaba, es decir, de la tercera sección de la primera parte de Ser y tiempo, conviene precisar que la pregunta por el sentido del ser en general, que orienta la analítica del Dasein, la ontología fundamental, señala al "tiempo" como el horizonte de toda comprensión del ser.

No parte aquí Heidegger de la conclusión de la segunda sección de Sein und Zeit, sino que se mueve en una orientación histórica. Desarrolla la cues-

\footnotetext{
${ }^{1}$ Aristóteles, Metafísica, z1, 1028-b2 sq.
} 
tión del ser y la analítica del Dasein en relación con la tradición occidental. En la primera parte y en el capítulo primero de la segunda parte la explicación de Zeit und Sein se plantea el problema del ser y la "diferencia ontológica" por primera vez.

En esta segunda parte del curso (verano de 1927) se propone tratar justamente lo que sería la tercera sección: Tiempo y ser. La pregunta ontológica fundamental por el sentido del ser en general. Las estructuras y "modos" (Weisen) fundamentales del ser.

La exposición de las cuatro tesis fundamentales a lo largo de la historia plantea la problemática de la ontología:

Primero: el de la diferencia ontológica, la diferencia del ser y el ente.

Segundo: el problema de la articulación fundamental del ser, la manera real y modo de ser del ente.

Tercero: el problema de las posibles modificaciones del ser y de la unidad del concepto ser en sus múltiples sentidos, Vieldeutigkeit.

Cuarto: el problema del carácter de verdad del ser.

Estos problemas fundamentales serían tratados en los cuatro capítulos de la segunda parte, pero en el texto publicado sólo está el capítulo primero. Encontramos la explicación de la temática de Zeit und Sein, "Tiempo y ser", así como los principios o fundamentos sistemáticos de la cuestión del ser en el pensar de Heidegger.

Capítulo primero:

El problema de la diferencia ontológica, se desarrolla en cuatro parágrafos.

19. Tiempo y temporalidad (Zeitlichkeit).

20. Temporalidad y Temporalität.

\section{Temporalität und Sein.}

22. Sein und Seiendes die ontologische Differenz (Ser y ente, la diferencia ontológica).

En la introducción expone su significación esencial. El problema de la diferencia del ser en general y del ente es lo primero. La explicación de esta diferencia hace posible investigar de manera expresa, clara y metódica, la diferencia entre ser y ente. Esto hará posible la consideración óntica del ente y el paso a la tematización ontológica del ser, a la posibilidad de la ontología, es decir, de la filosofía como ciencia, en el sentido de Hegel.

Se trata de algo prioritario. El ser y su diferencia del ente sólo se alcanza si entendemos al ser como tal y ante todo al ente a cuya estructura de ser pertenece la comprensión del ser, al Dasein. 
Se trata de la constitución fundamental del Dasein. De la analítica existenciaria, del fundamento, estructuras y unidad del Dasein. Aceptamos como bien fundada la analítica existenciaria expuesta en Sein und Zeit. La constitución ontológica del ser del Dasein se funda en la temporalidad (Zeitlichkeit).

¿Qué significa temporalidad?

Se puede partir de la comprensión vulgar del tiempo y descubrir su estructura fundamental.

La condición ontológica de la posibilidad de la comprensión del ser es la temporalidad de quien depende la ontología. Temporalität corresponde a Zeitlichkeit, pero sólo en la medida en que es condición de posibilidad de la comprensión del ser y de la ontología la llamamos Temporalität.

Zeitlichkeit expone, muestra, darstellt, el horizonte desde donde comprendemos al ser, la existencia (analítica existenciaria) se muestra como Temporalität, que constituye el horizonte para la comprensión del ser que esencialmente pertenece al Dasein.

Se trata de ver al ser en su determinabilidad y descubrir su problemática. Si el ser es visible en su determinación temporal, nos hallamos por ello en la posición en la que se capta claramente la diferencia entre ser y ente, y se establece el fundamento de la diferencia ontológica.

Para resolver la cuestión del ser no se puede considerar indispensable "completar" en una nueva edición Ser y tiempo. La tercera sección: el tiempo y el ser se expone en seminarios posteriores. Especialmente importante es este curso del semestre de verano de 1927, todavía en Marburgo, donde en la segunda parte expone con brevedad, pero rigurosa y claramente, el tiempo, la temporalidad, como condición necesaria para transitar a la ontología y a la comprensión de la diferencia ontológica del ser y del ente. Ésta es para Heidegger la tarea o el camino. Sobre todo el sentido y lo esencial de la ontología fundamental, es decir, de Sein und Zeit.

El ser y el origen del lenguaje, del "habla", de la palabra. El lenguaje y el hombre.

A lo largo de la historia de la Metafísica se determina el lenguaje a partir del logos ( $\lambda \circ \gamma_{\circ} \zeta$ ), como "proposición" (Aussage) y como conexión de representaciones.

Al hombre, "animal racional", le es dado el lenguaje. Hombre y expresión se determinan recíprocamente. El hombre como hombre es un ente, como todos los entes. Pero el hombre puede pertenecer y comprender al ser (seyn) desde su ser (Wesen) originario. El hombre "comprende" al ser, "cuida" la verdad del ser. "Pertenece" al ser en tanto que "alcanza" (Ereignete) el fundamento, la verdad del ser.

¿Cómo surge el lenguaje del ser? ¿Cómo aparece en el ser (Wesung des Seyns) el lenguaje? (metafísica-lenguaje-ser). 
El lenguaje surge del ser y pertenece al ser. El lenguaje es "nuestro" lenguaje como lenguaje materno y de nuestra historia. Nuestra historia, nosotros en nuestra relación con el ser.

La esencia corpórea, anímica-espiritual, del hombre se reconoce en el habla: 1) el lenguaje palabra-cuerpo, 2) el lenguaje alma (estado de ánimo, sentimientos, etcétera), 3) el lenguaje espíritu (lo pensado y representado), son determinaciones de toda filosofía del lenguaje. La esencia del lenguaje se constituye a partir de la determinación de la historia del ser del hombre. La tarea: ver el origen del lenguaje desde el ser (Wesung des Seyns).

Lenguaje y Ereignis. Sonido de la tierra, sonido (eco) del mundo. Lenguaje hablado o callado; primera y amplia humanización de los entes. Así parece. Pero es justo la originaria deshumanización del hombre como ente viviente y "sujeto" de todo lo anterior. Fundamentación del Dasein y de la posibilidad de deshumanización de los entes.

El lenguaje se funda en el silencio. El silencio es lo más oculto. Poesía y pensamiento se pertenecen. "Ninguna cosa sea donde falta la palabra".

Logos, Sage, el decir, la presencia. Decir y ser se pertenecen como palabra y cosa, pensar y poesía. El lenguaje no es una obra hecha, sino una actividad. Es labor del espíritu para capacitar al sonido articulado, para expresar el pensamiento. Lo esencial del lenguaje descansa en el habla.

No hay lenguaje "natural" de una naturaleza humana. Todo lenguaje es histórico (incluso si el hombre no conoce de historia). El lenguaje como información es histórico en el sentido y limitaciones de la época. Lo propio del lenguaje viene del habla (Sage).

El lenguaje es la casa del ser. Nuestra relación con el lenguaje se determina por la manera como pertenecemos al Ereignis (acontecimiento), al ser, a la totalidad, pero no en sentido metafísico.

Podemos, quizás, transformar nuestra relación con el lenguaje. Todo pensamiento "sensible" es poesía. Pero toda poesía es pensamiento.

En Beiträge zur Philosophie (vom Ereignis), "Contribuciones a la Filosofía (del acontecimiento)" tomo 65 de las Obras completas, publicado en 1989, y redactado en 1937-1938, desarrolla Heidegger el primer intento global, después de Ser y tiempo, de plantear de nuevo la cuestión del ser, en sentido histórico y originario. Se pregunta por el sentido de la verdad y del ser, es decir, de la manifestación del ser pensado como Ereignis. Se propone mostrar el tránsito de la metafísica al pensar histórico del ser que, como hemos dicho, está aún en camino. En esta obra fundamental se explica por qué, para Heidegger, no debe hablarse de "obra", sino de "camino".

La metafísica es la historia de Occidente y de su relación con la Tierra. Se inicia apenas el dominio incondicionado de la metafísica. Es una fatalidad del mundo europeo. 
Con el final de la metafísica se inicia lo que le era inaccesible, la aparición del ser del ente, aun cuando no se muestre la verdad del ser. El "acontecimiento", Ereignis, es el signo precursor y la primera manifestación de la verdad del ser. La superación de la metafísica sólo es posible a partir de la metafísica. Comienza la época de la metafísica acabada o su equivalente en la técnica planetaria. La metafísica de Nietzsche se inició en ese momento.

Parece tratarse del fin de la filosofía, pero no es de ningún modo el fin del pensar. Se abre la posibilidad de la verdad del ser. Nietzsche, en 1886, en sus notas a la última parte de Zaratustra dice: "Jugamos la carta de la verdad. La humanidad morirá quizás. Y bien, sea”. En Aurora escribió: "Lo que hay de nuevo en nuestra posición es la convicción, que no se ha dado en ninguna época, de que nosotros no poseemos la verdad. Los hombres de otro tiempo, incluso los escépticos, creían que poseían la verdad".

La filosofía en la época de la metafísica acabada se desarrolla sobre todo como antropología, física, bioética, psicología y así perece como metafísica. Predominan la técnica y la ausencia total de meditación. La técnica, forma suprema de la conciencia racional, y esta ausencia son lo mismo.

Si el origen del lenguaje lo encontramos en esta estructura del ser del Dasein y si el Dasein sólo puede comprenderse, para Heidegger, como momento o manifestación del ser, resulta clara la conocida definición del "lenguaje como morada del ser".

El lenguaje es lenguaje del ser. El ser del hombre implica la palabra. No es posible pensar al hombre sin el lenguaje. La palabra, el lenguaje, no se explica a partir de una concepción metafísica racionalista, ni tampoco a partir de una naturaleza humana. El lenguaje es necesariamente histórico, es necesariamente expresión del Dasein, del hombre en su desarrollo histórico.

El lenguaje implica necesariamente la temporalidad. Tiempo y ser en el plano de la ontología. Ser y tiempo todavía en la ontología fundamental. El ser del hombre es temporalidad y por lo tanto historia. Y éste es el fundamento último del lenguaje.

El lenguaje surge de la relación originaria del hombre con el mundo. Esta relación es anterior a todos los niveles en los que se plantean, tanto las concepciones tradicionales como las contemporáneas acerca del lenguaje.

El surgimiento del hombre es el surgimiento del lenguaje, así como también, según Heidegger, el surgimiento del pensar, de la poesía y de la religión. Todo pensamiento es poesía y toda poesía es pensamiento. En mundos separados, pero con este fondo común que es la palabra, que es tiempo y ser.

En Contribuciones a la filosofía (acontecimiento), encontramos el tránsito de la metafísica al pensamiento histórico del ser. Hay que intentar pensar la cuestión acerca de la verdad del ser. Incluso si hay buenos resultados, no 
puede caerse en el desarrollo de una obra del estilo antiguo de pensar. Lo que hay que pensar es el acontecimiento fundamental.

El pensamiento futuro es el camino del pensar a través del hasta ahora oculto dominio de la "presencia del ser", que apenas se ilumina y puede alcanzarse en su propio carácter "como acontecimiento". No se trata de presentar ya un ente sino de descubrir el ser en el "acontecimiento" y el devenir del "acontecer". Lo anterior implica de inmediato el cambio del ser del hombre desde la idea del "animal racional" hasta la del "ser-ahí". Este paso es lo que corresponde al Ereignis. El acontecer se muestra o transcurre en un habla pensante que corresponde al ser y se da en la palabra del ser.

Las Contribuciones preguntan en un camino que busca otro comienzo frente a la filosofía vigente. Este camino implica el tránsito a lo "abierto" de la historia y se desarrolla o funda como un "estar" duradero. Se trata del camino o del comienzo del otro pensar. El "decir" no depende del "habla", sino que es manifestación del ser.

El tiempo de los sistemas filosóficos ya pasó a la historia. Se trata ahora de desarrollar la búsqueda de la verdad del ser cuyo tiempo no ha llegado aún. Hay que buscar el principio del pensar, que será distinto y único a partir del primer comienzo.

La expresión máxima de la metafísica (ontoteología) es la teología que a lo largo de la historia de Occidente culmina en el Saber Absoluto y en la Voluntad de Poder como afirmación de lo finito.

La totalidad de los entes se da en el proceso de la negación y la negación de la negación. El ser es la "finitud", la estructura ontológica es finita. En Kant, el ser sería, en última instancia, lo finito en el hombre. Heidegger plantea en Ser y tiempo la finitud como fundamental y constitutiva. El ser es proyección o proceso. El surgimiento del ser-ahí es la única posibilidad de comprender la finitud, el tiempo y el ser. La ontología en la actualidad o las nuevas formas de pensar deberán ir más allá en esta dirección.

El ser es el fundamento, no del ente o del ente supremo en el sentido de la tradición, sino del aparecer en el ser-ahí y en la totalidad de lo finito. Pensar el ser es la única vía para superar la metafísica del ente supremo, así como su negación en el nihilismo. Tan "falso" es el nihilismo o la metafísica, como la afirmación o la negación de valores absolutos. Más difícil que la teología, el ente supremo o lo absoluto, es pensar el ser como "acontecimiento" y base o fundamento de la finitud. De todo lo que "es", del ser; el "ser-ahí" es el único capaz de "comprenderlo, fundarlo o expresarlo".

Las estructuras ontológicas del ser-ahí: habla, lenguaje, comprender, encontrarse, la muerte y la libertad, muestran la finitud y hacen posible que el hombre, el ser-ahí, pueda elevarse desde la ontología fundamental, "ser y tiempo", al ser como tiempo, como la finitud de todo ente. 
Frente a la metafísica, a su culminación en Hegel o en Nietzsche, hay que asumir la finitud y la negación como base del proceso que no terminaría en un "saber absoluto", sino que sería "la eterna negación" de todos los entes del mundo. El eterno retorno sería la repetición de la estructura de la finitud y de la manifestación del ser como "acontecimiento".

Como mostró Kant, es la finitud como estructura del hombre lo que permite fundar el conocimiento, la ética y la estética. Esto hace posible el desarrollo de una nueva metafísica y de la ontología. En lo más oculto y profundo de la razón humana se descubriría el ser como proceso y fundamento de todo lo finito. La totalidad sería necesariamente finita en tanto que manifestación del ser.

Ser y nada se muestran en la finitud. El ser es (acontecimiento) tiempo y finitud. El ente, todos los entes, son finitos en tanto que manifestaciones del ser.

Si hablamos del eterno retorno hay que entenderlo en el plano de lo finito, de lo cada vez necesariamente distinto y diferente. La totalidad, el ser, el espíritu, no es un ente absoluto, sino el proceso abierto de lo finito de todos los entes. A partir de Kant hay que entender el ser del hombre desde esta finitud. La totalidad sólo se manifiesta como proceso finito en el hombre.

Hay que comprender esta idea de la finitud como la estructura ontológica fundamental. En relación con Hegel no puede aceptarse el fin de la dialéctica o de la historia en el saber absoluto. En Hegel, el ser y la nada son fundamento de todo ente. En Marx, la dialéctica abierta, la negación lleva al hombre real a la naturaleza y a la historia. El ser se manifiesta, "acontece" en el Dasein, en el lenguaje y por tanto en los entes. Incluso en la religión, el ente supremo es afirmación y negación del ente. Se manifiesta como fundamento y finitud de todo ente. El ser es y se manifiesta como negación y finitud. Es lo finito. El ser y el no ser del ente son el fundamento, "acontecimiento", devenir o historia, tiempo, proceso de la negación (Hegel).

¿Cuáles son los caminos o métodos para la filosofía en nuestra época? Hemos planteado una de las vías posibles o fundamentales: la ontología como el camino de la libertad frente a lo establecido o desarrollado en las corrientes o disciplinas científico-técnicas, filosóficas o ético-políticas vigentes. ¿Por qué libertad? Porque es el fundamento olvidado del pensar y del hacer, a partir del cual hay que construir nuevas posibilidades de desarrollo y afirmación del ser humano. El fin de la metafísica, el nihilismo, la muerte de Dios, la crisis de todos los valores, señalan la urgencia de nuevas formas y métodos del pensar.

Hay un "aparente" desarrollo ligado al proceso de la ciencia y de la técnica. Pero se trata, en la mayor parte de las corrientes filosóficas o de las "obras personales", de un pensamiento auxiliar o complementario al servicio del proceso histórico real. No quisiera, ni creo necesario mencionar estas "teo- 
rías", que en lugar de orientar o dirigir se convierten en comentaristas o analistas de la ciencia, la moral y la política.

Frente a la crisis, hay que volver a concepciones o ideas fundamentales, como base o referencia crítica para la búsqueda de nuevos caminos: ante todo recuperar el sentido originario del pensar filosófico. Recordemos no solo el origen en Grecia o la gran tradición de Occidente sino también a grandes filósofos modernos. Kant, culminación y punto de partida del pensamiento moderno y contemporáneo, especialmente en el campo de la ética y la política donde la "dignidad" del hombre es principio y fin de la acción. Su idea o principio elemental del pensar es válida:

1. Pensar por cuenta propia.

2. Ponerse en lugar de otros.

3. Ser coherente, congruente.

En la filosofía, dice Kant, lo fundamental son tres cuestiones que se resumen en una final:

1. Was kann ich wissen? (¿Qué puedo saber?)

2. Was soll ich tun? (¿Qué debo hacer?)

3. Was darf ich hoffen? (¿Qué puedo esperar?)

4. Was ist der Mensch? (¿Qué es el hombre?)

En Hegel, culminan la metafísica y la filosofía de occidente. Afirma la racionalidad, la universalidad y la concepción de la dialéctica, que con la de Marx ofrece caminos fundamentales en nuestros días.

La racionalidad y la dialéctica en Hegel y en Marx:

1. Conocimiento de principios universales.

2. La seriedad de la vida pletórica que se adentra en la experiencia de la cosa misma.

3. El concepto se descubre y penetra en la profundidad de la cosa.

A partir de las críticas de Kant se abre en el Juicio reflexionante el camino al método heurístico, con base también en la dialéctica trascendental, en las ideas regulativas, en la fenomenología y en la hermenéutica. Nietzsche, el nihilismo y el pensar, la crítica a los valores y la historia de Occidente abren también posibilidades esenciales. En suma, hay caminos para el pensar de nuestros días. Tanto en el campo de la ontología como en los teóricos y prácticos del pensamiento contemporáneo hay vías o métodos fundamentales. En especial en la ética y la política, en el arte y en la ciencia. 
La heurística como reflexión y orientación: "Ideas regulativas" o "Juicio reflexionante".

La dialéctica en el campo de la historia y el análisis político como reflexión acerca de la metafísica y su posible superación en una nueva concepción del hombre y del ser.

Nuestra conclusión última es la reafirmación de la libertad como fundamento, no sólo de la práctica (ética, política, histórica), sino de la teoría, es decir, de la ciencia y de la técnica, del arte y de la poesía. 